\title{
Dual Blockade of the Homeostatic Chemokine CXCL12 and the Proinflammatory Chemokine CCL2 Has Additive Protective Effects on Diabetic Kidney
} Disease

Murthy N. Darisipudi, ${ }^{*}$ Onkar P. Kulkarni, ${ }^{*}$ Sufyan G. Sayyed, ${ }^{*}$ Mi Ryu, ${ }^{*}$ Adriana Migliorini, ${ }^{*}$ Costanza Sagrinati, ${ }^{\dagger}$ Eliana Parente, ${ }^{\dagger}$ Axel Vater, ${ }^{\ddagger}$ Dirk Eulberg, ${ }^{\ddagger}$ Sven Klussmann, ${ }^{\ddagger}$

Paola Romagnani, ${ }^{\dagger}$ and Hans-Joachim Anders*

From the Nephrological Center," Medical Policlinic, University of Munich, Munich, Germany; the Excellence Centre for Research, ${ }^{\dagger}$ Transfer and High Education for the Development of De Novo Therapies (DENOTHE), University of Florence, Florence, Italy; and NOXXON Pharma $A G,{ }^{\ddagger}$ Berlin, Germany

Monocyte/ chemoattractant protein-1/chemokine ligand (CCL) 2 and stromal cell-derived factor-1/ CXCL12 both contribute to glomerulosclerosis in mice with type 2 diabetes mellitus, through different mechanisms. CCL2 mediates macrophage-related inflammation, whereas CXCL12 contributes to podocyte loss. Therefore, we hypothesized that dual antagonism of these chemokines might have additive protective effects on the progression of diabetic nephropathy. We used chemokine antagonists based on structured L-enantiomeric RNA (so-called Spiegelmers) ie, the CCL2-specific mNOX-E36 and the CXCL12-specific NOX-A12. Male $\mathrm{db} / \mathrm{db}$ mice, uninephrectomized at the age of 6 weeks, received injections of Spiegelmer, both Spiegelmers, nonfunctional control Spiegelmer, or vehicle from the age of 4 months for 8 weeks. Dual blockade was significantly more effective than monotherapy in preventing glomerulosclerosis. CCL2 blockade reduced glomerular leukocyte counts and renal-inducible nitric oxide synthase or IL-6 mRNA expression. CXCL12 blockade maintained podocyte numbers and renal nephrin and podocin mRNA expression. Consistently, CXCL12 blockade suppressed nephrin mRNA up-regulation in primary cultures of human glomerular progenitors induced to differentiate toward the podocyte lineage. All previously mentioned parameters were significantly improved in the dual-blockade group, which also suppressed proteinuria and was associated with the highest levels of glomerular filtration rate. Blood glucose levels and body weight were identical in all treatment groups. Dual chemokine blockade can have additive effects on the progression of diabetic kidney disease when the respective chemokine targets mediate different pathomechanisms of disease (ie, inflammation and progenitor differentiation toward the podocyte lineage). (Am J Pathol 2011, 179:116-124; DOI: 10.1016/j.ajpath.2011.03.004)

Diabetic nephropathy (DN) is a leading cause of chronic kidney disease. ${ }^{1-3}$ Novel treatment strategies are necessary because the current concept of angiotensin blockade and blood pressure control cannot prevent disease progression in all cases. ${ }^{4}$ In DN, the glomerular tuft undergoes a slow but progressive structural remodeling characterized by glomerular hypertrophy, diffuse and nodular accumulation of extracellular mesangial matrix, and podocyte damage. ${ }^{5}$ The latter is thought to account for the progression of microalbuminuria from early stages to overt proteinuria and glomerulosclerosis at late-stage $\mathrm{DN}$. The onset and progression of DN involves numerous additional pathomechanisms, including the deposition of advanced glycation end products, endothelial dysfunction, and increased local expression of growth factors and proinflammatory mediators. ${ }^{6}$ Most chemokines belong to the latter group of factors because proinflamma-

Supported by the Else Kröner-Fresenius Stiftung and the EU Integrated Project "INNOCHEM" (FP6-518167 to H.-J.A.).

Accepted for publication March 21, 2011.

Disclosures: A.V., D.E., and S.K. are employees of NOXXON Pharma $A G$, which supplied the oligonucleotides.

M.N.D. prepared parts of this article as a doctoral thesis at the Faculty of Medicine, University of Munich

Address reprint request to Hans-Joachim Anders, M.D., Medizinische Poliklinik, Klinikum der Universität München-Innenstadt, Pettenkoferstrasse 8a, 80336 Munich, Germany. E-mail: hjanders@med.unimuenchen.de. 
tory chemokines promote tissue inflammation and remodeling by recruiting and activating immune cells in DN, as in other types of kidney diseases. ${ }^{7-10}$ For example, targeted deletion or inhibition of monocyte chemoattractant protein-1 [renamed chemokine ligand (CCL) 2] signaling can prevent glomerulosclerosis by blocking macrophage recruitment to the glomeruli of mice with type 1 or 2 diabetes mellitus. ${ }^{11,12}$ In fact, CCL2 may represent a promising therapeutic target in DN because delayed onset of CCL2 blockade was able to prevent diabetic glomerulosclerosis and restore the glomerular filtration rate (GFR) by preventing glomerular macrophage recruitment in late-stage DN of uninephrectomized $\mathrm{db} / \mathrm{db}$ mice with type 2 diabetes. ${ }^{13}$

Although structurally related, a subgroup of the chemokine superfamily, known as "homeostatic" chemokines, displays functions independent of tissue inflammation. Homeostatic chemokines are rather constitutively expressed because they contribute to the physiological homing and migration of immune cells in the bone marrow or lymphoid organs. ${ }^{14}$ For example, recently, the homeostatic chemokine stromal cell-derived factor-1, also named CXCL12, was constitutively expressed by podocytes and CXCL12 blockade prevents diabetic glomerulosclerosis in a way that was independent of glomerular macrophage recruitment. ${ }^{15}$ The mechanism underlying the protective effect of CXCL12 blockade on DN remains unclear, but a profound effect on podocyte counts and proteinuria was documented. Because we could not detect increased bone marrow-derived progenitor cells in kidneys of treated $\mathrm{db} / \mathrm{db}$ mice, an enhancer effect on the capacity of intraglomerular podocyte progenitors to restore podocytes in DN was suspected. ${ }^{15}$

Our previous attempts to increase the therapeutic effects of chemokine blockade on the progression of DN were not successful. For example, blockade of CCR2 and CCR5 with a dual antagonist did not reveal any additional effects compared with single CCR blockade (S.G.S and H.J.A., unpublished data). We had attributed this finding to the fact that CCR2 and CCR5 are redundant when coexpressed on the same class of monocytes, which may preclude additive effects on glomerular pathological characteristics in DN.

Therefore, we hypothesized that combining the protective effects of reducing glomerular leukocyte recruitment by blocking the proinflammatory chemokine CCL2 with the protective effects on podocyte loss by blocking the homeostatic chemokine CXCL12 should finally elicit additive protective effects on diabetic glomerulosclerosis. As such, we compared monotherapy of either CCL2 or CXCL12 antagonists with dual blockade in an accelerated model of glomerulosclerosis in $\mathrm{db} / \mathrm{db}$ mice with type 2 diabetes.

\section{Materials and Methods}

\section{CCL2 and CXCL12 Antagonistic Spiegelmers}

The sequences of the CCL2 antagonistic Spiegelmer mNOX-E36 (5'-GGCGACAUUGGUUGGGCAUGAGGC-
GAGGCCCUUUGAUGAAUCCGCGGCCA-3') and the CXCL12 antagonistic Spiegelmer NOX-A12 (5'-GCGUGGUGUGAUCUAGAUGUAUUGGCUGAUCCUAGUCAGGUACGC-3') were obtained from in vitro selection experiments that were essentially performed as described. ${ }^{16}$ To distinguish possible target-specific from unspecific substance class effects, a nonfunctional Spiegelmer with the reverse sequence of NOX-A12 (5'-CGCAUGGACUGAUCCUAGUCGGUUAUUAGAUCUAGUGUGGUGCG-3') was used. The Spiegelmers were modified with 40-kDaba branched polyethylene glycol at the $3^{\prime}$ end (mNOX-E36) or the $5^{\prime}$ end (NOX-A12 and reverse sequence of NOX-A12). mNOX-E36 and NOX-A12 bind to CCL2 and CXCL12, respectively, with subnanomolar affinities. Chemokine inhibition was determined for each Spiegelmer using leukocyte chemotaxis assays, as described. ${ }^{16,17}$ All Spiegelmers were previously tested as single agents in DN by our group, as described. ${ }^{13}$

\section{Animal Studies}

Male diabetic C57BLKS db/db mice or nondiabetic C57BL/6 mice, aged 5 weeks, were obtained from Taconic (Ry, Denmark) and housed in filter-top cages with a 12-hour dark-light cycle. All animals had unlimited access to food and water throughout the duration of the study. At the age of 6 weeks, uninephrectomy ( $1 \mathrm{~K}$ mice) or sham surgery was performed through a $1-\mathrm{cm}$ flank incision, as previously described. ${ }^{18}$ In the sham surgery group, the kidney was left in situ. At the age of 4 months, $1 \mathrm{~K} \mathrm{db} / \mathrm{db}$ mice with documented blood glucose levels $>11 \mathrm{mmol} / \mathrm{L}$ and creatinine/albumin ratios $>3$ (ratio in age-matched wild-type mice, 0.1 ) were divided into four groups ( $n=10$ to 12); each of these groups received either nil (no injections) or s.c. injections of $50 \mathrm{mg} / \mathrm{kg}$ NOX-A12, mNOX-E36, or control Spiegelmer in 5\% glucose or $5 \%$ glucose itself as a vehicle control, on every other day. This dose corresponds to $13.4 \mathrm{mg} / \mathrm{kg}$ NOXA12 or $14.4 \mathrm{mg} / \mathrm{kg}$ mNOX-A36 if based on the oligonucleotide part of the molecule as anhydrous free acid. The treatment dose and injection intervals were comparable to those in previous $\mathrm{db} / \mathrm{db}$ mouse studies, ${ }^{13,15,19}$ with similar formulations of Spiegelmers targeting these chemokines. Treatment was continued for 8 weeks. Tissues were harvested for histopathological evaluation at the end of the treatment period, 3 to 4 hours after the last Spiegelmer injection. Blood and urine samples were obtained at monthly intervals for the estimations of urinary albumin, by enzyme-linked immunosorbent assay (Bethyl Labs, Montgomery, TX); and serum and urinary creatinine, by Jaffé reaction (DiaSys Diagnostic Systems, Holzheim, Germany). Blood glucose levels were monitored using an Accu check sensor (Roche, Mannheim, Germany). Plasma chemokine levels were determined at the end of the treatment period by enzyme-linked immunosorbent assay (CCL2: BD Biosciences Pharmingen, San Diego, CA; CXCL12: R\&D Systems, Wiesbaden, Germany). All animal experiments were approved by the local government authorities. 


\section{Histopathological Characteristics}

Kidneys were harvested from each mouse and were fixed in $10 \%$ formalin in PBS and embedded in paraffin. Sections, 2 $\mu \mathrm{m}$, were stained with PAS reagent. Glomerular sclerotic lesions were assessed using a semiquantitative score by a blinded observer, as follows: 0 indicates no lesion; 1, <25\% sclerotic; $2,25 \%$ to $49 \%$ sclerotic; $3,50 \%$ to $74 \%$ sclerotic; and $4,75 \%$ to $100 \%$ sclerotic. Fifteen glomeruli were analyzed per section. The number of glomerular cells was determined on PAS stains by counting cell nuclei in 15 glomerular tufts per section. Pole-cutted glomerular cross sections were ignored. Immunostaining was performed, as described, ${ }^{20}$ using the following primary antibodies: CD45 (leukocytes; BD Biosciences, Mannheim; 1:10), Wilms tumor (WT)-1 (podocytes; Santa Cruz, Santa Cruz, CA; 1:200), Ki-67 (proliferating cells; Dianova, Hamburg, Germany; 1:50), terminal deoxynucleotidyl transferase-mediated dUTP nick-end labeling (TUNEL; apoptotic cells; Roche, Mannheim; 1:10). TUNEL- and DAPI-positive cells (Sigma-Aldrich, Steinheim, Germany) were counted by fluorescent microscopy, and all other stains were assessed by light microscopy in 15 glomeruli per section by a blinded observer (H.J.M.).

\section{In Vitro Studies}

Normal renal tissues were obtained from four patients who underwent nephrectomy because of renal cell carcinoma, in agreement with the Ethical Committee on Human Experimentation of the Azienda Ospedaliero-Universitaria Careggi, Florence, Italy. Human embryonic kidney 293 cells were purchased from American Type Culture Collection (Manassas, VA) and cultured as specified by the supplier. The human keratinocyte cell line was a gift from Dr. Rosa Mancina (Florence, Italy). Human renal progenitors were obtained by immunomagnetic sorting for CD133 after depletion of digested total renal cell suspensions for CD45 and podocalyxin, as previously described. ${ }^{18}$ Primary cultures of human renal progenitors were $>99 \%$ positive for CD133 and CD24 coexpression, as checked by fluorescence-activated cell sorter analysis. Podocyte markers were absent. For podocyte differentiation, cells were treated for 2 days with VRAD medium, as previously described. ${ }^{18,21}$ Nephrin mRNA quantification was performed using Assay on Demand kits (Applied Biosystems, Warrington, UK), ${ }^{20,21}$ according to the manufacturer's recommendations; and Taq-Man RT-PCR was performed using a $7900 \mathrm{HT}$ Real Time PCR System (Applied Biosystems), as previously described. ${ }^{20,21}$ To accurately compare samples by real-time RT-PCR, an identical number of cells $(n=10,000)$ was analyzed in all experiments; and glyceraldehyde-3-phoshate dehydrogenase was used to evaluate mRNA quality and integrity.

\section{RNA Preparation and Real-Time Quantitative (TaqMan) RT-PCR}

Total RNA was isolated from kidneys using a Qiagen RNA extraction kit (Hilden, Germany) following the manufacturer's instructions. After quantification, RNA quality was assessed using agarose gels. From isolated RNA, cDNA was prepared using reverse transcriptase (Superscript II; Invitrogen, Carlsbad, CA). Real-time PCR was performed using SYBR Green PCR master mix, and results were analyzed with a Light Cycler 480 (Roche). All gene expression values were normalized using 18s RNA as a housekeeping gene. All primers used for amplification were from Metabion (Martinsried, Germany): CCL2 (sense, 5'-CCTGCTGTTCACAGTTGCC-3'; antisense, 5'-ATTGGGATCATCTTGCTGGT-3'), CXCL12 (sense, 5'-TTTCAGATGCTTGACGTTGG-3'; antisense, 5'-GCGCTCTGCATCAGTGAC-3'), tumor necrosis factor (sense, 5'-AGGGTCTGGGCCATAGAACT-3'; antisense, 5'-CCACCACGCTCTTCTGTCTAC-3'), inducible nitric oxide synthase (sense, 5'-TGAAGAAAACCCCTTGTGCT-3'; antisense, 5'-TTCTGTGCTGTCCCAGTGAG-3'), nephrin (sense, 5'-CTCTTTCTACCGCCTCAACG-3'; antisense, 5'-TTAGCAGACACGGACACAGG-3'), podocin (sense, 5'-CAGGAAGCAGATGTCCCAGT-3'; antisense, 5'-TGACGTTCCCTTTTTCCATC-3'), IL-6 (sense, 5'-ACCAGAGGAAATTTTCAATAGGC-3'; antisense, 5'-TGATGCACTTGCAGAAAACA-3'), and 18s RNA (sense, 5'-AGGGCCTCACTAAACCATCC-3'; antisense, 5'-GCAATTATTCCCCATGAACG-3').

\section{Statistical Analysis}

Data are presented as mean \pm SEM. A comparison of groups was performed using an analysis of variance, and post hoc Bonferroni's correction was used for multiple comparisons. $P<0.05$ indicated statistical significance.

\section{Results}

\section{Dual CCL2-CXCL12 Blockade Has Additive Effects on Glomerulosclerosis in $d b / d b$ Mice}

To test our hypothesis, we initiated s.c. injections with vehicle alone, control Spiegelmer, mNOX-E36 (anti-CCL2 Spiegelmer), NOX-A12 (anti-CXCL12 Spiegelmer), or a combination of the latter two at a dose of $50 \mathrm{mg} / \mathrm{kg}$ given every other day from the age of 4 to 6 months in uninephrectomized $\mathrm{db} / \mathrm{db}$ mice. To ensure Spiegelmer exposure and its biological activity in vivo, we determined plasma CCL2 and CXCL12 levels at the beginning and the end of the study. Plasma CCL2 and CXCL12 levels remained undetectable or low in sham-operated on $\mathrm{db} / \mathrm{db}$ mice and vehicle- or control Spiegelmer-treated $1 \mathrm{~K} \mathrm{db} / \mathrm{db}$ mice. Anti-CXCL12 or anti-CCL2 Spiegelmer injections increased the plasma levels of either chemokine in $1 \mathrm{~K} \mathrm{db} / \mathrm{db}$ mice (Figure 1). This finding is consistent with previous observations ${ }^{13,15,17}$ with these Spiegelmers, indicating that Spiegelmer antagonists retain their molecular targets in the circulation. Such Spiegelmerchemokine complexes no longer display chemotactic activity in trans-well migration assays (data not shown). Body weight and blood glucose levels did not differ among the injected $1 \mathrm{~K} \mathrm{db} / \mathrm{db}$ mice groups (Figure 2). Renal histomorphological features in 6-month-old $\mathrm{db} / \mathrm{db}$ mice showed moderate glomerulosclerosis compared 


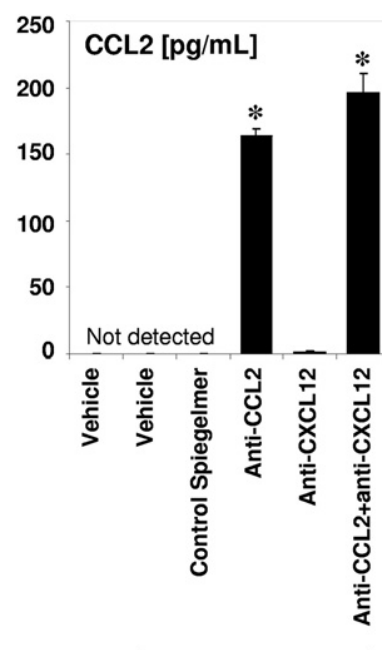

14

24 weeks

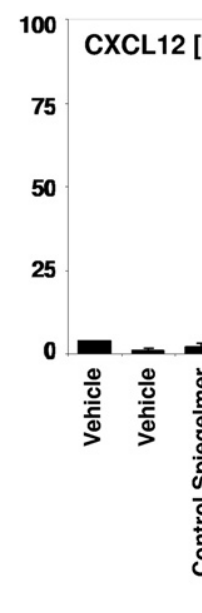

14

\section{4 weeks}

Figure 1. Plasma chemokine levels in Spiegelmer-treated $\mathrm{db} / \mathrm{db}$ mice Plasma CCL2 and CXCL12 levels were determined in 14- and 24-week-old uninephrectomized $\mathrm{db} / \mathrm{db}$ mice by enzyme-linked immunosorbent assay, which represents before and after treatment. Data are given as mean \pm SEM ${ }^{*} P<0.001$ versus the 24 -week vehicle group.

with age-matched nondiabetic mice that underwent sham surgery, which was aggravated to diffuse glomerulosclerosis by early uninephrectomy of $\mathrm{db} / \mathrm{db}$ mice (Figure 3). CCL2 and CXCL12 inhibition reduced the extent of glomerulosclerosis in uninephrectomized $\mathrm{db} / \mathrm{db}$ mice to the level of age-matched sham-operated on $\mathrm{db} / \mathrm{db}$ mice, whereas the control Spiegelmer had no effect. Dual CCL2-CXCL12 blockade further improved glomerular pathological features, with significantly less severe lesions and more normal glomeruli compared with either of the monotherapies. $1 \mathrm{~K} \mathrm{db} / \mathrm{db}$ mice with dual blockade displayed even less glomerular pathological features than age-matched $\mathrm{db} / \mathrm{db}$ mice that underwent sham surgery (Figure 3B). Thus, dual CCL2-CXCL12 blockade has additive protective effects on glomerulosclerosis in $1 \mathrm{~K}$ $\mathrm{db} / \mathrm{db}$ mice.

\section{Only CCL2 Blockade Reduces Leukocyte Numbers in Glomeruli of $1 \mathrm{~K} d b / d b$ Mice}

Chemokine-mediated glomerular pathological features in $\mathrm{db} / \mathrm{db}$ mice can be mediated by leukocyte recruitment. ${ }^{11,13}$ Therefore, we evaluated the number of glomerular leukocytes by immunostaining for CD45. Significant numbers of $\mathrm{CD}_{4} 5^{+}$leukocytes were detected in glomeruli of vehicle- or control Spiegelmer-treated $1 \mathrm{~K} \mathrm{db} / \mathrm{db}$ mice (Figure 4). Consistent with previous data, ${ }^{13,15}$ the numbers of glomerular CD45 ${ }^{+}$leukocytes were significantly reduced by CCL2, but not by CXCL12, blockade. The numbers of glomerular leukocytes were also reduced accordingly with dual-chemokine blockade (Figure 4). Therefore, blockade of the proinflammatory chemokine CCL2, but not of the homeostatic chemokine CXCLI2, affects the number of glomerular leukocytes in $\mathrm{db} / \mathrm{db}$ mice.

\section{Dual CCL2-CXCL12 Blockade Has Additive Effects on Podocyte Numbers in db/db Mice}

Previously, CXCL12 blockade protected $1 \mathrm{~K} \mathrm{db} / \mathrm{db}$ mice from podocyte loss as another marker of glomerular pathological features in $\mathrm{db} / \mathrm{db}$ mice. ${ }^{15}$ We questioned whether dual-chemokine blockade would have additive protective effects on podocytes and quantified glomerular podocytes by WT-1 immunostaining in all groups of $1 \mathrm{~K}$ $\mathrm{db} / \mathrm{db}$ mice. Compared with an average number of 15 to 20 WT-1-positive podocytes in murine glomerular cross sections (data not shown), the 6-month-old $1 \mathrm{~K} \mathrm{db} / \mathrm{db}$ mice revealed only an average of 11 cells per glomerular cross section. CCL2 and, particularly, CXCL12 blockade both significantly increased glomerular podocyte counts (Figure 5). Interestingly, dual CCL2-CXCL12 blockade showed a small, but statistically significant, additive effect up to an average of 17 WT-1-positive cells per glomerular cross section (Figure 5). Thus, dual CCL2CXCL12 inhibition improves glomerular pathological features in association with more podocytes in $\mathrm{db} / \mathrm{db}$ mice.
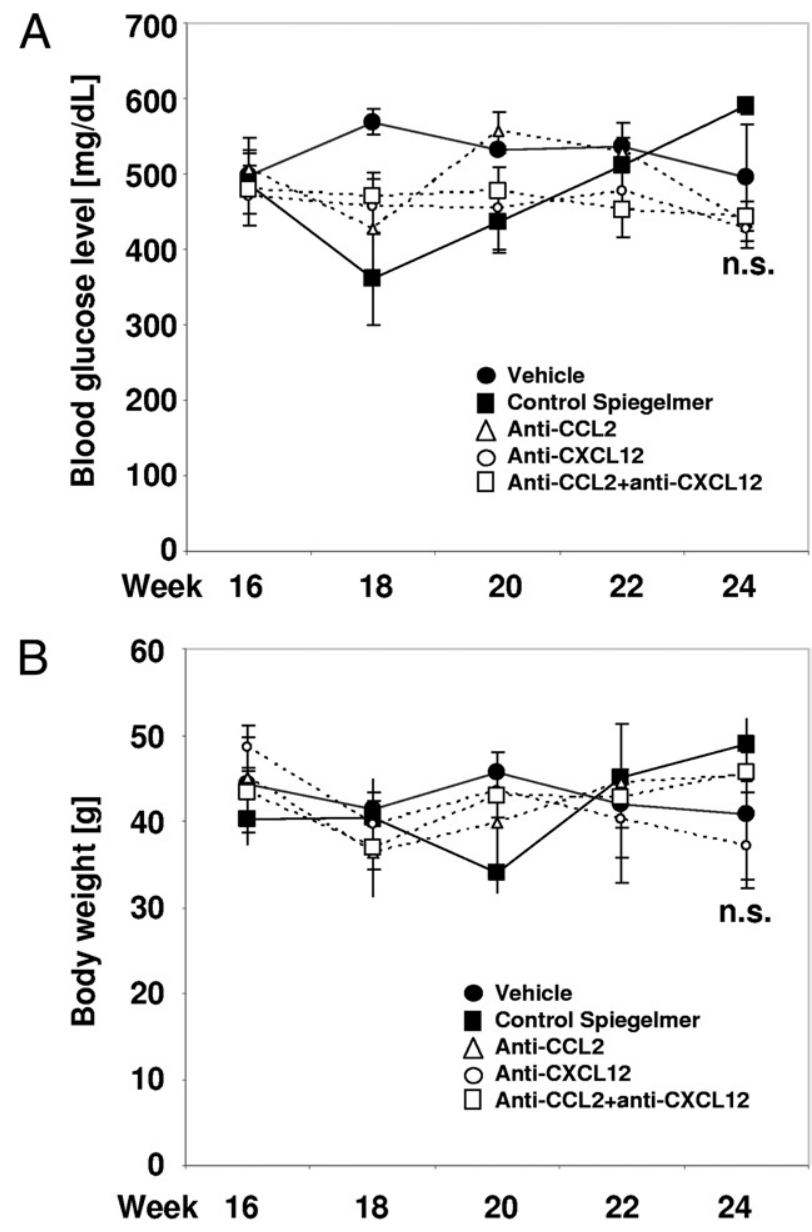

Figure 2. Blood glucose levels and body weight in uninephrectomized $\mathrm{db} / \mathrm{db}$ mice. Uninephrectomized $\mathrm{db} / \mathrm{db}$ mice with different treatments were monitored for blood glucose levels (A) and body weight (B) from baseline (at the age of 16 weeks) until the end of the study (at the age of 24 weeks). Data are given as the mean \pm SEM. n.s. indicates nonsignificant. 
A
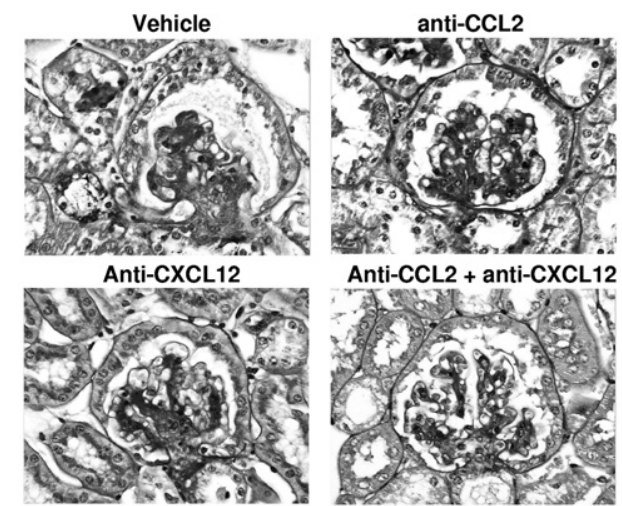

$\mathrm{B}$

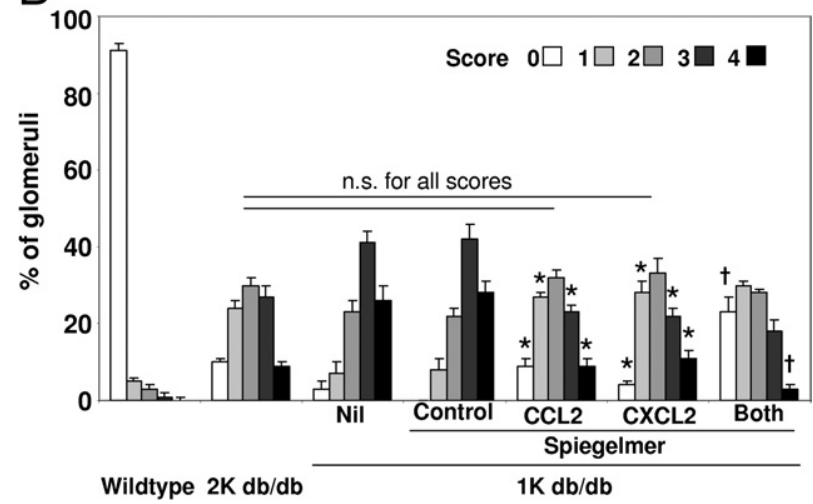

Figure 3. Renal pathological features in 6-month-old $\mathrm{db} / \mathrm{db}$ mice. A: Renal sections from $1 \mathrm{~K}$ mice of the different treatment groups were stained with PAS. Stains show representative glomeruli from each group. Original magnification, $\times 400$. B: PAS stains were scored from 0 to 4 for the extent of glomerulosclerosis, as described. From each mouse, 15 glomeruli from each renal section were graded by that score. The graph illustrates the mean \pm SEM percentage of each score from all mice in each group. Uninephrectomy was associated with a shift toward higher scores of glomerulosclerosis, as seen in the control Spiegelmer- and vehicletreated $1 \mathrm{~K} \mathrm{db} / \mathrm{db}$ mice. Single blockade with either anti $(\alpha)$-chemokine Spiegelmer significantly reduced the overall scores compared with control Spiegelmer-treated $1 \mathrm{~K} \mathrm{db} / \mathrm{db}$ mice. ${ }^{*} P<0.05$. Moreover, dual blockade further significantly reduced the percentage of glomeruli, with a score of 4 , and even increased the percentage of glomeruli, with a score of 1 , compared with either single chemokine blockade. ${ }^{\dagger} P<0.05$. n.s. indicates nonsignificant.

\section{Dual CCL2-CXCL12 Blockade and Renal mRNA Expression in db/db Mice}

Next, we determined the mRNA expression levels of leukocyte- and podocyte-related genes in kidneys of $1 \mathrm{~K}$ $\mathrm{db} / \mathrm{db}$ mice. Both antagonists did not significantly alter intrarenal expression of their target gene (Figure 6). However, CCL2 blockade, but not CXCL12 blockade, was associated with lower mRNA levels of inducible nitric oxide synthase, a parameter of activated macrophages (Figure 6). The cytokine IL-6 showed a trend toward lower levels in all treatment groups. In contrast, CXCL12 blockade increased the mRNA levels of nephrin and podocin, two podocyte parameters, much more than CCL2 blockade. Dual-chemokine blockade increased these two podocyte markers most significantly (Figure 6). Together, CCL2 and CXCL12 single blockade has different effects on renal mRNA expression profiles; both effects combine with dual blockade.

\section{Dual CCL2-CXCL12 Blockade and Glomerular Cell Counts in $d b / d b$ Mice}

Glomerular pathological features are often related to an altered cell turnover of glomerular cells. Therefore, we determined the total numbers of glomerular cells, Ki-67 ${ }^{+}$ proliferating glomerular cells, and TUNEL ${ }^{+}$apoptotic glomerular cells. Total numbers of glomerular cells were determined by counting DAPI-positive nuclei in glomerular tuft cross sections. None of the interventions significantly affect the total number of cell nuclei in glomerular tufts (Figure 7A). The overall numbers of $\mathrm{Ki}-67^{+}$proliferating cells within glomeruli were low, but CCL2 and CXCL12 blockade both significantly reduced their numbers in glomeruli (Figure 7B). Dual CCL2-CXCL12 blockade had an additive suppressive effect on glomerular cell proliferation (Figure 7B). TUNEL ${ }^{+}$apoptotic cells were only occasionally seen and did not differ between all treatment groups (data not shown). We conclude that dual-chemokine blockade has an additive antiproliferative effect on glomerular cells in type 2 diabetic mice; however, given the opposite effects (eg, on glomerular podocyte counts), the total number of glomerular cells was not significantly affected.
A

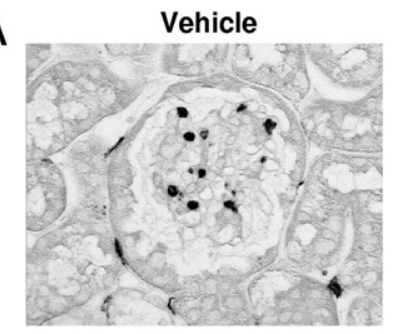

Anti-CXCL12

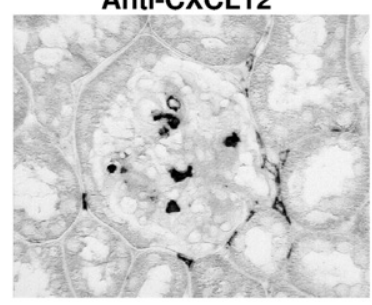

B

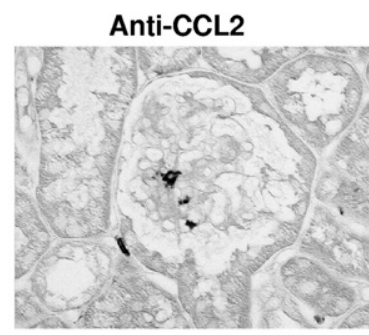

Anti-CCL2 + Anti-CXCL12

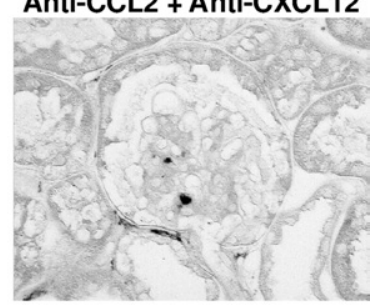

Glom. CD45+ cells

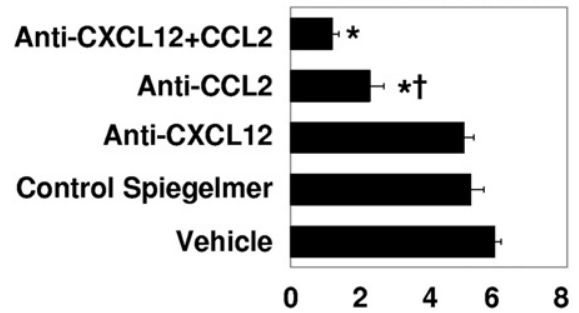

Figure 4. Glomerular leukocyte infiltrates in $1 \mathrm{~K} \mathrm{db} / \mathrm{db}$ mice. A: Renal sections from $1 \mathrm{~K}$ mice of all treatment groups were stained for CD 45 . Original magnification, $\times 400$. B: The graph shows the mean \pm SEM CD $45^{+}$cells in 15 glomeruli (Glom.) in sections from 6 -month-old $1 \mathrm{~K} \mathrm{db} / \mathrm{db}$ mice of each group. Only anti-CCL2 Spiegelmer affected the number of glomerular CD $45^{+}$ cells. ${ }^{*} P<0.01$ versus control Spiegelmer; ${ }^{\dagger} P<0.01$ versus both monotherapies. 


\section{Dual CCL2-CXCL12 Blockade Increases GFR and Reduces Proteinuria in $d b / d b$ Mice}

We determined the GFR by assessing fluorescein isothiocyanate insulin clearance kinetics in all groups of mice. Uninephrectomy was associated with a reduced GFR compared with a normal GFR of approximately $350 \mathrm{~mL}$ in mice. Either CCL2 or CXCL12 blockade significantly increased GFR in 6-month-old 1K db/db mice (Figure 8A). Dual CCL2-CXCL12 blockade was associated with the highest GFR (Figure 8A). Albuminuria is another clinically important parameter of glomerular dysfunction and damage. We compared urinary albumin/creatinine ratios at the beginning and at the end of the treatment course in all groups. CCL2 and CXCL12 significantly reduced the urinary albumin/creatinine ratio when compared with control Spiegelmer treatment at 6 months (Figure 8B). When compared with the baseline urinary albumin/creatinine ratio, CXCL12 and dual blockade most effectively prevented proteinuria. Therefore, we conclude that dual CCL2-CXCL12 blockade has a potent protective effect on GFR decline. CXCL12 blockade, either alone or in combination, effectively prevents proteinuria in $1 \mathrm{~K} \mathrm{db} / \mathrm{db}$ mice, which is consistent with its positive effect on podocytes.

A

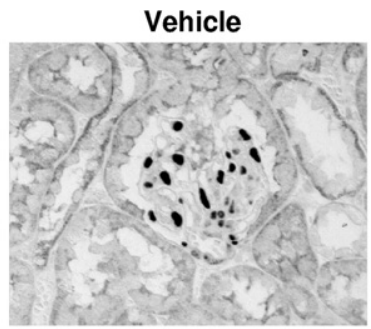

Anti-CXCL12

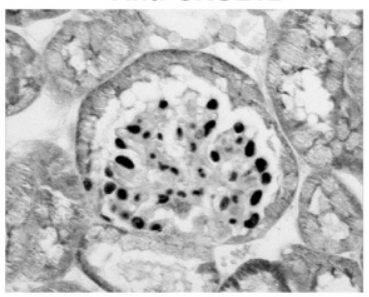

B

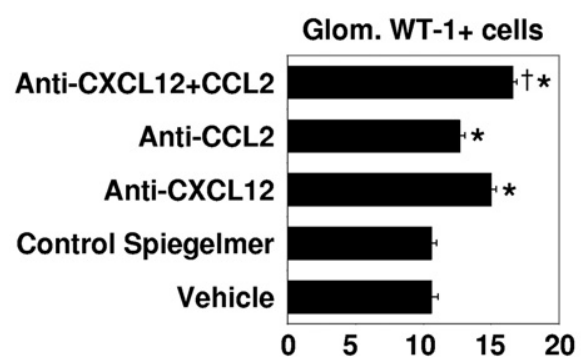

Figure 5. Podocyte numbers in $1 \mathrm{~K} \mathrm{db} / \mathrm{db}$ mice. A: Renal sections from $1 \mathrm{~K}$ mice of all treatment groups were stained for WT-1. Original magnification, $\times 400$. B: The graph shows the mean \pm SEM WT-1-positive cells in 15 glomeruli (Glom.) in sections from 6-month-old $1 \mathrm{~K} \mathrm{db} / \mathrm{db}$ mice of each group. There was a potent effect of the anti-CXCL12 Spiegelmer on the number of podocytes and an additive effect of dual blockade versus antiCXCL12 monotherapy. ${ }^{*} P<0.05$ versus control Spiegelmer; ${ }^{\dagger} P<0.05$ versus both monotherapies.
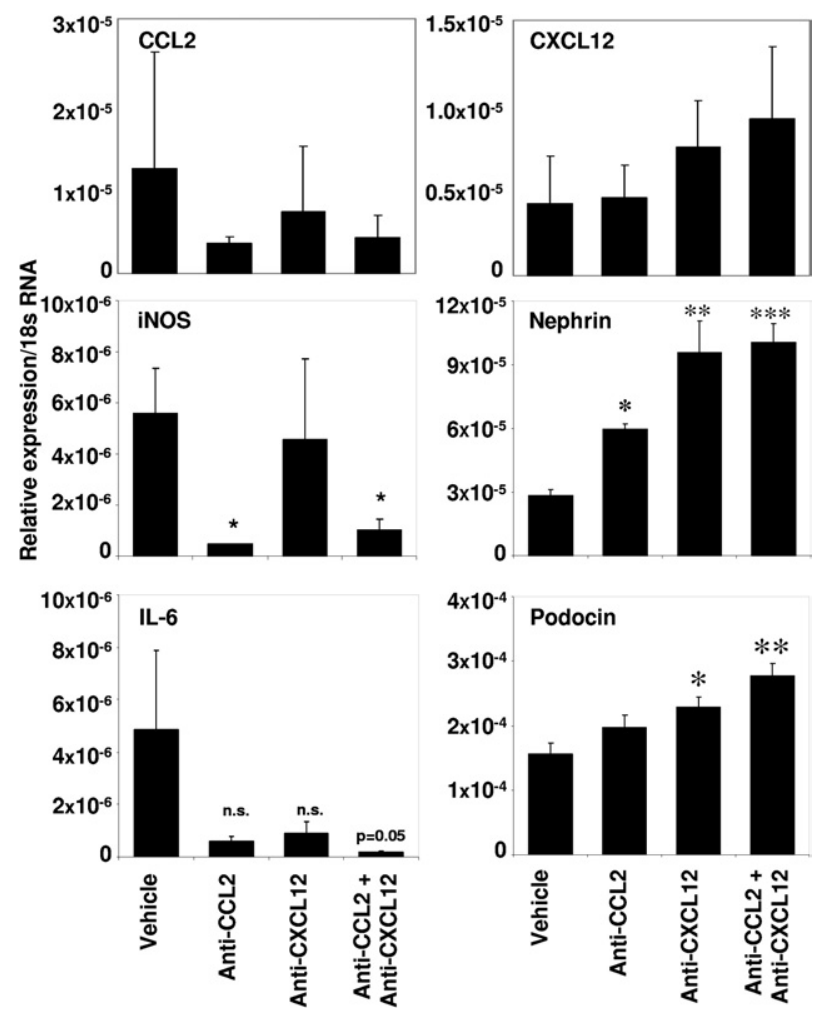

Figure 6. Renal mRNA expression in $1 \mathrm{~K} \mathrm{db} / \mathrm{db}$ mice. RNA isolates from kidneys of $1 \mathrm{~K} \mathrm{db} / \mathrm{db}$ mice underwent quantitative real-time PCR for several genes, as indicated. Data are expressed as mean \pm SEM of the ratio of the specific mRNA versus that of 18 s ribosomal RNA. ${ }^{*} P<0.05$, ${ }^{* *} P<0.01$, and ${ }_{* * * *} P<0.001$ versus vehicle. n.s. indicates nonsignificant.

\section{CXCL12 Suppresses Nephrin de Novo Expression of Podocyte Progenitors}

How does CXCL12 blockade significantly increase podocyte numbers and nephrin expression without any direct effect on podocytes, which we previously excluded? ${ }^{15}$ Given the critical role of CXCL12 in the maintenance of
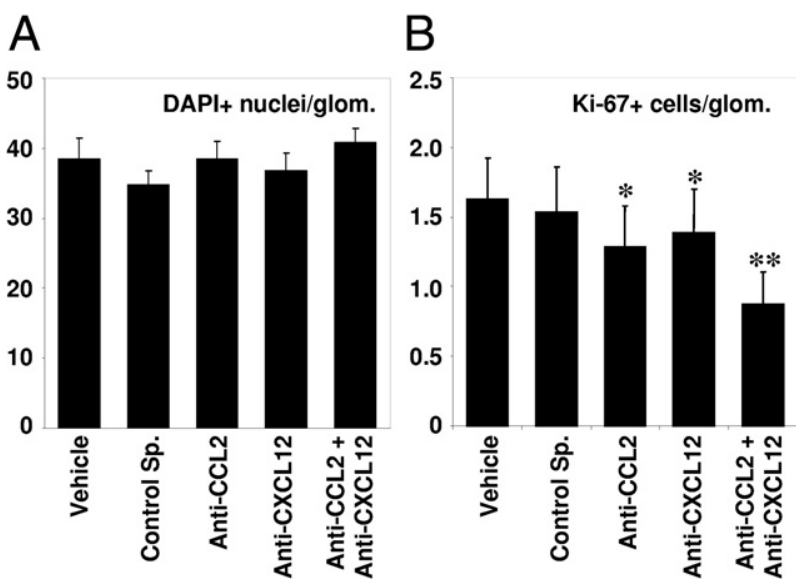

Figure 7. Proliferation of glomerular (glom.) cells in $1 \mathrm{~K} \mathrm{db} / \mathrm{db}$ mice. A: The number of $\mathrm{DAPI}^{+}$cell nuclei was counted in renal sections from mice of all groups, as indicated. B: Renal sections from mice of all groups were stained for the proliferation marker Ki-67. The graphs show the mean \pm SEM positive cells in 15 glomeruli in sections from 6 -month-old $1 \mathrm{~K} \mathrm{db} / \mathrm{db}$ mice of each group. ${ }^{*} P<0.05,{ }^{* *} P<0.01$ versus control Spiegelmer treatment. 
A

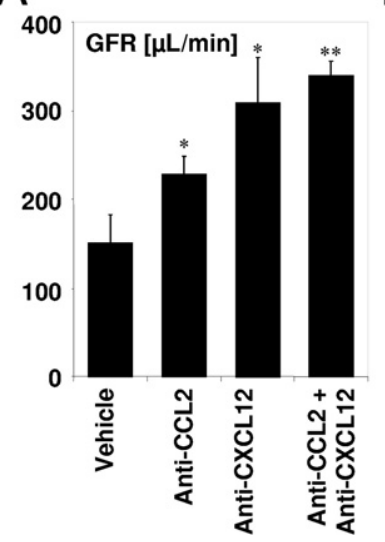

$\mathrm{B}_{25}$

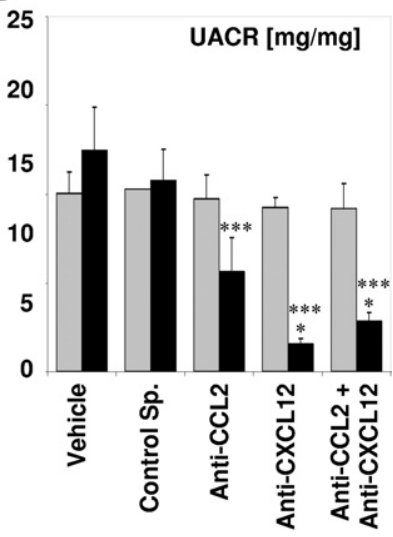

Figure 8. GFR and albuminuria in 6-month-old $\mathrm{db} / \mathrm{db}$ mice. A: GFR was determined by fluorescein isothiocyanate-insulin clearance kinetics in all groups at the end of the study, as described in the Materials and Methods. $1 \mathrm{~K}$ mice treated with dual-chemokine blockade have the GFR levels. B: Urinary albumin/creatinine ratios (UACRs) were determined as a functional marker of the glomerular filtration barrier at the initiation ( 4 months, gray bars) and termination ( 6 months, black bars) of treatment. Data are given as the mean \pm SEM from at least six mice in each group. ${ }^{*} P<0.05,{ }^{* * *} P<0.01$ versus control-Spiegelmer-treated $1 \mathrm{~K} \mathrm{db} / \mathrm{db}$ mice; ${ }^{* * *} P<0.05$ versus baseline UACR in the respective group (gray bar).

stem cell niches in multiorgan systems, we evaluated the possibility that podocyte-derived CXCL12 may regulate the potential of renal progenitor cells to differentiate toward podocytes. Several groups ${ }^{15-19}$ have recently reported that a subpopulation of parietal epithelial cells in glomeruli represents progenitor cells and has the capacity to differentiate into mature podocytes by progressively migrating and differentiating from the urinary pole of the Bowman's capsule toward the glomerular tuft. Therefore, we questioned whether CXCL12 serves as a factor that regulates the differentiation of renal progenitor cells toward the podocyte phenotype. A previous report ${ }^{15}$ indicated that culturing human renal progenitors in the VRAD medium over 2 days resulted in their differentiation into podocytes, as demonstrated by novel expression of nephrin, WT-1, synaptopodin, podocin, and anti-glomerular epithelial protein 1 at both the mRNA and protein levels. Because CXCL12 blockade up-regulated nephrin expression in our in vivo models, in this study, we evaluated primary cultures of human renal progenitors that are already known to express receptors for $\mathrm{CXCL} 12^{15}$ for their potential to up-regulate nephrin mRNA expression in the presence or absence of this chemokine. The human embryonic kidney epithelial cell line 293 and the human keratinocyte cell line were evaluated as additional controls for nephrin mRNA expression before and after treatment with VRAD. As shown in Figure 9A, the effect of VRAD treatment was irrelevant in human embryonic kidney 293 and human keratinocyte cell lines, whereas it induced strong up-regulation of nephrin mRNA expression in primary cultures of human renal progenitors, as assessed by quantitative RT-PCR. Adding recombinant CXCL12 suppressed nephrin mRNA up-regulation in a dose-dependent manner (Figure 9A). When CXCL12 was blocked with anti-CXCL12 Spiegelmer, de novo mRNA expression of nephrin was no longer compromised, an

effect that was not observed with the unspecific control Spiegelmer (Figure 9B). Thus, CXCL12 suppresses the potential of renal progenitors to express nephrin. Vice versa, CXCL12 antagonism increased levels of nephrin mRNA expression, which may explain the findings on CXCL12 blockade in $\mathrm{db} / \mathrm{db}$ mice.

\section{Discussion}

We hypothesized that dual blockade of CCL2 and CXCL12 was superior compared with single blockade in preventing the progression of diabetic glomerulosclerosis because both approaches target different disease pathomechanisms. Our data support this hypothesis and demonstrate that dual blockade combines the protective effect on CCL2-mediated glomerular leukocyte recruitment with that on CXCL12-mediated loss of podocytes. Thus, our data render dual CCL2-CXCL12 blockade as a novel strategy to more efficiently prevent glomerulosclerosis in type 2 diabetes.

Data of the present study confirm previous reports ${ }^{13,15}$ on CCL2 blockade with Spiegelmers in the same experimental setup. Attempts to increase the therapeutic benefit by initiating treatment earlier were not successful. ${ }^{19}$ In contrast, even a later onset of anti-CCL2 Spiegelmer treatment (from the age of 5 months) was equally effective in preventing glomerulosclerosis in $1 \mathrm{~K} \mathrm{db} / \mathrm{db}$ mice. ${ }^{19}$ In the present and in previous studies, CCL2 blockade

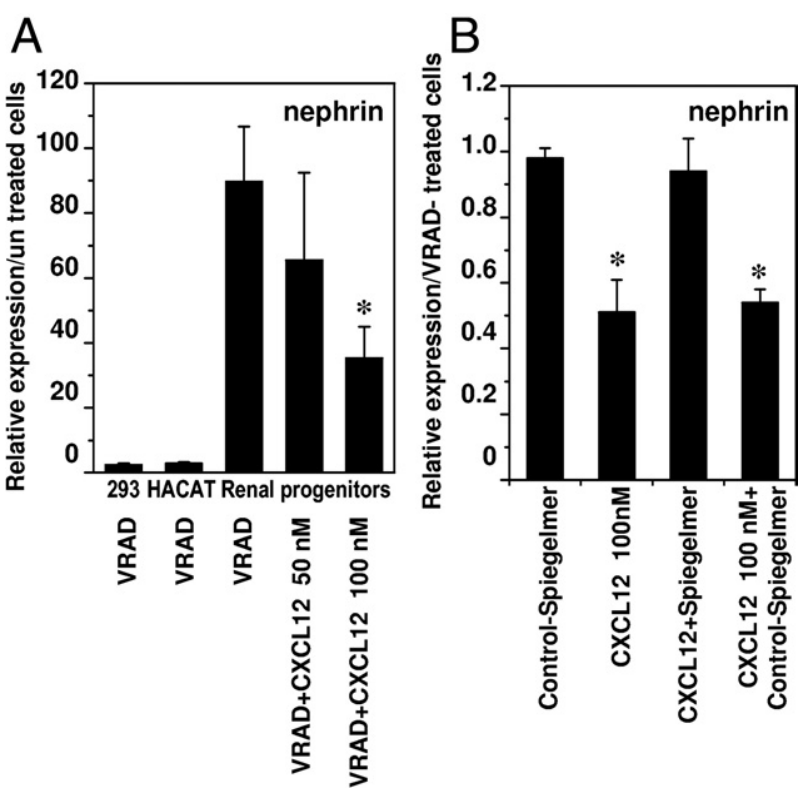

Figure 9. CXCL12 and de novo nephrin expression in renal progenitor cells A: Human embryonic kidney 293 cells, human keratinocyte cells (HACAT), and human renal progenitors were cultured in VRAD medium, as described in the Materials and Methods. Data are expressed as fold increase versus the respective untreated cell type, as assessed in an identical number of cells. On human renal progenitors, recombinant CXCL12 was added at different concentrations, as indicated, and nephrin mRNA expression was determined by real-time RT-PCR. B: Cells were cultured as before, plus adding anti-CXCL12 or control Spiegelmer. Only anti-CXCL12 Spiegelmer prevented the CXCL12mediated suppression of de novo nephrin mRNA expression. Data are expressed as fold increase versus VRAD-treated renal progenitors (B), as assessed in an identical number of cells. Data are given as mean \pm SEM. ${ }^{*} P<$ 0.05 
resulted in a $50 \%$ to $60 \%$ reduction of glomerular leukocytes that entirely represent macrophages in the $\mathrm{db} / \mathrm{db}$ mouse model. ${ }^{21}$ These findings are consistent with data from models of types 1 and 2 diabetes in Ccl2-deficient mice ${ }^{11,12}$ or with CCR2 blockade in $1 \mathrm{~K} \mathrm{db} / \mathrm{db}$ mice. ${ }^{22}$ $\mathrm{CCR}^{+}$macrophages belong to the proinflammatory (M1) phenotype that contributes to intrarenal inflammation and tissue damage via the release of reactive oxygen species or cytokines, such as tumor necrosis factor$\alpha .{ }^{23,24}$ Thus, anti-CCL2-Spiegelmer-treated $1 \mathrm{~K} \mathrm{db} / \mathrm{db}$ mice revealed fewer glomerular leukocytes that were associated with lower intrarenal mRNA levels of inducible nitric oxide synthase, IL-6, or tumor necrosis factor- $\alpha$ at the end of the study. Consistent with previous findings, the protection from glomerulosclerosis with CCL2 blockade correlated with some lower levels of proteinuria and a higher GFR. Thus, CCL2 blockade prevents the progression of DN by interfering with macrophage-driven glomerular inflammation.

The present study also reconfirms previous data ${ }^{15}$ on CXCL12 blockade in this model. NOX-A12 prevented the progression of glomerulosclerosis in $1 \mathrm{~K} \mathrm{db} / \mathrm{db}$ mice without affecting glomerular leukocyte counts, indicating that the homeostatic chemokine CXCL12 is not involved in glomerular leukocyte recruitment. Recently, CXCL12 was constitutively expressed in podocytes. ${ }^{15} \mathrm{CXCL} 12$ blockade also increased podocyte numbers in $1 \mathrm{~K} \mathrm{db} / \mathrm{db}$ mice in the present study. Furthermore, CXCL12 blockade increased renal mRNA expression levels of nephrin and podocin, two podocyte-slit membrane-related proteins that serve as markers of podocyte differentiation. ${ }^{25}$ Podocyte damage is an important pathomechanism promoting the progression of diabetic glomerulosclerosis and seems to result from various triggers that foster podocyte dedifferentiation and podocyte apoptosis. ${ }^{26}$ It remains unclear whether the beneficial effect of CXCL12 blockade on podocyte numbers relates to a protective effect on podocyte death or detachment or is the result of enhanced podocyte regeneration from local podocyte progenitors (eg, from parietal epithelial cells). ${ }^{27,28}$ The latter concept may be more likely because CXCL12 blockade also mobilizes hematopoietic progenitor cells from the bone marrow. ${ }^{29-31}$ In fact, our in vitro studies demonstrate that CXCL12 suppressed the induced maturation of renal progenitors toward podocytes, as documented by inhibition of de novo nephrin expression. This effect was specific to renal progenitors and was not observed in other epithelial cells. Our in vitro finding that CXCL12 blockade reverts this inhibitory effect on nephrin expression may correspond to our in vivo observation that CXCL12 blockade increased renal nephrin mRNA expression and podocyte numbers. Thus, CXCL12 blockade may enhance renal progenitor differentiation toward the podocyte lineage, thus enhancing podocyte regeneration.

Thus, one hypothesis is that dual blockade with antiCCL2- and anti-CXCL12-Spiegelmers could lead to Spiegelmer-Spiegelmer interaction effects, such as complex formation or additive off-target effects. However, the plasma chemokine level analysis confirmed that dual blockade does not significantly affect the biological ac- tivity of each Spiegelmer in terms of binding its natural target (in the plasma). This observation is consistent with the finding that $1 \mathrm{~K} \mathrm{db} / \mathrm{db}$ mice with dual blockade recapitulate the biological effects of single blockade. For example, $1 \mathrm{~K} \mathrm{db} / \mathrm{db}$ mice treated with dual blockade displayed the reduction of glomerular leukocytes and a trend toward lower inducible nitric oxide synthase and tumor necrosis factor- $\alpha$ expression to the same extent as seen with CCL2 blockade. In addition, dual blockade also increased podocyte numbers and nephrin or podocin mRNA expression to the same extent as seen with CXCL12 blockade only. As a result, the combination of these different ways dual blockade was more potent than single blockade in preventing diffuse glomerulosclerosis was evidenced by significantly fewer glomeruli with global glomerulosclerosis and significantly more normal glomeruli. This additive therapeutic effect was less prominent for GFR and proteinuria because CXCL12 blockade by itself already had a profound effect on these two functional end points. Together, these data first document that chemokine antagonist combinations hold a potential for additive preventive effects on (diabetic) glomerulosclerosis when the individual chemokine targets mediate different pathomechanisms in the specific disease process (ie, inflammation and renal progenitor differentiation toward the podocyte lineage).

\section{Acknowledgments}

We thank Dan Draganovici and Jana Mandelbaum for their expert technical support and Stefan Vonhoff and the chemistry group of NOXXON Pharma AG for providing the oligonucleotides.

\section{References}

1. Zimmet $P$, Alberti KG, Shaw J: Global and societal implications of the diabetes epidemic. Nature 2001, 414:782-787

2. Ritz E, Rychlik I, Locatelli F, Halimi S: End-stage renal failure in type 2 diabetes: a medical catastrophe of worldwide dimensions. Am J Kidney Dis 1999, 34:795-808

3. II: incidence and prevalence of ESRD. Am J Kidney Dis 1999, 34(Suppl 1):S40-S50

4. Svensson M, Sundkvist G, Arnqvist HJ, Bjork E, Blohme G, Bolinder J, Henricsson M, Nystrom L, Torffvit O, Waernbaum I, Ostman J, Eriksson JW: Signs of nephropathy may occur early in young adults with diabetes despite modern diabetes management: results from the nationwide population-based Diabetes Incidence Study in Sweden (DISS). Diabetes Care 2003, 26:2903-2909

5. Qian Y, Feldman E, Pennathur S, Kretzler M, Brosius FC 3rd: From fibrosis to sclerosis: mechanisms of glomerulosclerosis in diabetic nephropathy. Diabetes 2008, 57:1439-1445

6. Yamagishi S, Fukami K, Ueda S, Okuda S: Molecular mechanisms of diabetic nephropathy and its therapeutic intervention. Curr Drug Targets 2007, 8:952-959

7. Ruster $\mathrm{C}$, Wolf $\mathrm{G}$ : The role of chemokines and chemokine receptors in diabetic nephropathy. Front Biosci 2008, 13:944-955

8. Galkina E, Ley K: Leukocyte recruitment and vascular injury in diabetic nephropathy. J Am Soc Nephrol 2006, 17:368-377

9. Tesch GH: MCP-1/CCL2: a new diagnostic marker and therapeutic target for progressive renal injury in diabetic nephropathy. Am J Physiol Renal Physiol 2008, 294:F697-F701

10. Vielhauer V, Kulkarni O, Reichel CA, Anders HJ: Targeting the recruitment of monocytes and macrophages in renal disease. Semin Nephrol 2010, 30:318-333 
11. Chow FY, Nikolic-Paterson DJ, Ma FY, Ozols E, Rollins BJ, Tesch GH: Monocyte chemoattractant protein-1-induced tissue inflammation is critical for the development of renal injury but not type 2 diabetes in obese db/db mice. Diabetologia 2007, 50:471-480

12. Chow FY, Nikolic-Paterson DJ, Ozols E, Atkins RC, Rollin BJ, Tesch $\mathrm{GH}$ : Monocyte chemoattractant protein-1 promotes the development of diabetic renal injury in streptozotocin-treated mice. Kidney Int 2006, 69:73-80

13. Ninichuk V, Clauss S, Kulkarni O, Schmid H, Segerer S, Radomska E, Eulberg D, Buchner K, Selve N, Klussmann S, Anders HJ: Late onset of Ccl2 blockade with the Spiegelmer mNOX-E36-3'PEG prevents glomerulosclerosis and improves glomerular filtration rate in $\mathrm{db} / \mathrm{db}$ mice. Am J Pathol 2008, 172:628-637

14. Moser B, Wolf M, Walz A, Loetscher P: Chemokines: multiple levels of leukocyte migration control. Trends Immunol 2004, 25:75-84

15. Sayyed SG, Hagele H, Kulkarni OP, Endlich K, Segerer S, Eulberg D, Klussmann S, Anders HJ: Podocytes produce homeostatic chemokine stromal cell-derived factor-1/CXCL12, which contributes to glomerulosclerosis, podocyte loss and albuminuria in a mouse model of type 2 diabetes. Diabetologia 2009, 52:2445-2454

16. Eulberg D, Purschke W, Anders HJ, Selve N, Klussmann S: Spiegelmer NOX-E36 for renal diseases. Therapeutic Oligonucleotides. Edited by J Kurreck. Biomolecular Sciences Series of the Royal Society of Chemistry (RSC), Cambridge, UK. 2008, pp 200-221

17. Kulkarni O, Pawar RD, Purschke W, Eulberg D, Selve N, Buchner K, Ninichuk V, Segerer S, Vielhauer V, Klussmann S, Anders HJ: Spiegelmer inhibition of CCL2/MCP-1 ameliorates lupus nephritis in MRL-(Fas)Ipr mice. J Am Soc Nephrol 2007, 18:2350-2358

18. Ninichuk V, Khandoga AG, Segerer S, Loetscher P, Schlapbach A Revesz L, Feifel R, Khandoga A, Krombach F, Nelson PJ, Schlondorff $\mathrm{D}$, Anders $\mathrm{HJ}$ : The role of interstitial macrophages in nephropathy of type 2 diabetic db/db mice. Am J Pathol 2007, 170:1267-1276

19. Sayyed SG, Gaikwad AB, Lichtnekert J, Kulkarni O, Eulberg D Klussmann S, Tikoo K, Anders HJ: Progressive glomerulosclerosis in type 2 diabetes is associated with renal histone $\mathrm{H} 3 \mathrm{~K} 9$ and $\mathrm{H} 3 \mathrm{~K} 23$ acetylation: H3K4 dimethylation and phosphorylation at serine 10. Nephrol Dial Transplant 2010, 25:1811-1817

20. Patole PS, Schubert S, Hildinger K, Khandoga S, Khandoga A, Segerer S, Henger A, Kretzler M, Werner M, Krombach F, Schlondorff $\mathrm{D}$, Anders HJ: Toll-like receptor-4: renal cells and bone marrow cells signal for neutrophil recruitment during pyelonephritis. Kidney Int 2005, 68:2582-2587

21. Chow F, Ozols E, Nikolic-Paterson DJ, Atkins RC, Tesch GH: Macrophages in mouse type 2 diabetic nephropathy: correlation with diabetic state and progressive renal injury. Kidney Int 2004, 65:116-128

22. Sayyed SG, Ryu M, Kulkarni OP, Schmid H, Lichtnekert J, Grüner S, Green L, Mattei P, Hartmann G, Anders HJ: Oral treatment with a chemokine receptor CCR2 antagonist prevents glomerulosclerosis and renal failure in type 2 diabetes. Kidney Int 2011, in press

23. Mantovani A, Sica A, Sozzani S, Allavena P, Vecchi A, Locati M: The chemokine system in diverse forms of macrophage activation and polarization. Trends Immunol 2004, 25:677-686

24. Ricardo SD, van Goor H, Eddy AA: Macrophage diversity in renal injury and repair. J Clin Invest 2008, 118:3522-3530

25. Patrakka J, Tryggvason K: New insights into the role of podocytes in proteinuria. Nat Rev Nephrol 2009, 5:463-468

26. Susztak K, Raff AC, Schiffer M, Bottinger EP: Glucose-induced reactive oxygen species cause apoptosis of podocytes and podocyte depletion at the onset of diabetic nephropathy. Diabetes 2006, 55: 225-233

27. Appel D, Kershaw DB, Smeets B, Yuan G, Fuss A, Frye B, Elger M, Kriz W, Floege J, Moeller MJ: Recruitment of podocytes from glomerular parietal epithelial cells. J Am Soc Nephrol 2009, 20:333-343

28. Ronconi E, Sagrinati C, Angelotti ML, Lazzeri E, Mazzinghi B, Ballerini L, Parente E, Becherucci F, Gacci M, Carini M, Maggi E, Serio M, Vannelli GB, Lasagni L, Romagnani S, Romagnani P: Regeneration of glomerular podocytes by human renal progenitors. J Am Soc Nephro 2009, 20:322-332

29. Peled A, Petit I, Kollet O, Magid M, Ponomaryov T, Byk T, Nagler A, Ben-Hur H, Many A, Shultz L, Lider O, Alon R, Zipori D, Lapidot T: Dependence of human stem cell engraftment and repopulation of NOD/SCID mice on CXCR4. Science 1999, 283:845-848

30. Avecilla ST, Hattori K, Heissig B, Tejada R, Liao F, Shido K, Jin DK, Dias S, Zhang F, Hartman TE, Hackett NR, Crystal RG, Witte L, Hicklin DJ, Bohlen P, Eaton D, Lyden D, de Sauvage F, Rafii S: Chemokinemediated interaction of hematopoietic progenitors with the bone marrow vascular niche is required for thrombopoiesis. Nat Med 2004 10:64-71

31. Sugiyama T, Kohara H, Noda M, Nagasawa T: Maintenance of the hematopoietic stem cell pool by CXCL12-CXCR4 chemokine signaling in bone marrow stromal cell niches. Immunity 2006, 25:977-988 